\title{
Efeitos do Tratamento com Alarme para Enurese no Autoconceito de Crianças
}

\author{
Rafaela Almeida Ferrari, Rodrigo Fernando Pereira, Felipe Alckmin-Carvalho', \\ Renatha Rafihi-Ferreira, Edwiges Ferreira de Mattos Silvares \\ Universidade de São Paulo, São Paulo-SP, Brasil
}

\section{RESUMO}

O objetivo do estudo foi avaliar a eficácia do tratamento comportamental para enurese com uso de alarme, realizado à distância, e avaliar efeitos da intervenção no autoconceito dos participantes. Trata-se de um ensaio clínico com delineamento quase-experimental de grupos. Trinta e uma crianças com enurese primária monossintomática participaram ( $M($ idade $)=8,3 ; D P=1,3), 15$ meninos (48,3\%). Para avaliar a enurese, foi utilizado o Formulário de Avaliação da Enurese; para o autoconceito, a Escala de Autoconceito Infantojuvenil; para instruções sobre o tratamento, o Guia de Pais. A taxa de sucesso no tratamento foi de $71 \%$. Houve diferença estatisticamente detectável na variação do autoconceito pessoal pré e pós-intervenção $(M=4,8 ; D P=1,9 ;$ vs $M=6,1 ; D P=1,9 ; p=0,01)$, independentemente do desfecho, e diferenças no autoconceito social entre as médias de crianças que tiveram sucesso no tratamento $(M=9,8 ; D P=1,9$ vs $M=0,5 ; D P=1,5 ; p=0,03)$. A proposta de tratamento da enurese apresentada é viável e eficaz e impactou positivamente o autoconceito das crianças avaliadas.

Palavras-chave: enurese; tratamento comportamental; ensaio clínico; autoconceito.

\section{ABSTRACT - Effects of Alarm Treatment for Enuresis on Children's Self-Concept}

The objective of the study was to evaluate the efficacy of enuresis alarm therapy, performed at a distance, and to evaluate the effects of the intervention on the participants' self-concept. This is a clinical trial with a quasi-experimental group design. Thirty-one children with mono-symptomatic primary enuresis participated $(M(\mathrm{age})=8.3, S D=1.3), 15$ boys $(48.3 \%)$. Assessments were made using the Enuresis Evaluation Form; Child-Juvenile Self-Concept Scale; and the Parents' Guide, for treatment instructions. Treatment success rate was $71 \%$. There was a statistically significant difference in the variation of the personal self-concept before and after intervention $(M=4.8, S D=1.9 ;$ v $M=6.1, S D=1.9 ; p=0.01)$, regardless of the outcome $(M=9.8, S D=1.9$ vs $M=0.5, S D=1.5 ; p=0.03)$, and differences in the social self-concept among the means of children who had successful treatment $(M=9.8, S D=1.9 v s=0.5, D P=1.5$; $p=0.03$ ). The proposed enuresis treatment is feasible and effective, and positively impacted the self-concept of the children evaluated. Keywords: enuresis; bedwetting; alarm therapy; behavioral treatment; clinical trial; self-concept.

\section{RESUMEN - Efectos del Tratamiento con Alarma para Enuresis en el Autoconcepto de Niños}

El objetivo del estudio fue evaluar la eficacia del tratamiento comportamental para Enuresis, con uso de alarma, realizado a distancia, y evaluar también los efectos de la intervención en el autoconcepto de los participantes.Se trata de un ensayo clínico con delineamiento casi experimental de grupos. Participaron 31 niños con enuresis primaria monosintomático ( $M$ (edad)=8,3; $D P=1,3), 15$ varones $(48,3 \%)$. Para evaluar la enuresis fue utilizado el Formulario de Evaluación de Enuresis, para el autoconcepto; Escala de Autoconcepto Infanto Juvenil, para instrucciones sobre el tratamiento; Guía de Padres. La tasa de éxito del tratamiento fue de $71 \%$. Hubo diferencia estadísticamente detectable en la variación del autoconcepto personal antes y después de la intervención $(M=4,8 ; D P=1,9 ; v s M=6,1$; $D P=1,9 ; p=0,01)$ independiente de los resultados, y diferencias en el autoconcepto social entre el promedio de los niños que tuvieron un tratamiento exitoso $(M=9,8 ; D P=1,9 v s M=0,5 ; D P=1,5 ; p=0,03)$. La propuesta del tratamiento de enuresis presentada es viable y eficaz e impactó positivamente en el autoconcepto de los niños evaluados.

Palabras clave: enuresis; tratamiento comportamental; ensayo clínico; autoconcepto.

OManual de Diagnóstico e Estatística de Transtornos Mentais (DSM-V) (American Psychiatric Association, 2013) aponta os seguintes critérios para o diagnóstico de enurese: eliminação de urina durante o dia ou à noite, na cama ou nas roupas; episódios que ocorrerem no mínimo duas vezes por semana, por pelo menos três meses consecutivos, ou que produzirem sofrimento ou prejuízo significativo no funcionamento social, acadêmico, ou em outras áreas importantes na vida do indivíduo; idade cronológica (ou mental) de, no mínimo, cinco anos, e incontinência urinária não atribuída aos efeitos fisiológicos diretos de uma substância ou condição médica geral. 
Além dos critérios estabelecidos pela quinta edição do DSM, há outro bastante difundido entre os clínicos e pesquisadores, desenvolvido pela International Children's Continence Society (ICCS), no qual uma criança com idade superior a cinco anos apresenta enurese quando há micção normal em local ou hora inadequada (Nevéus et al., 2006). Os autores estabelecem uma frequência de, pelo menos, uma vez ao mês. Além disso, apontam que a quantidade de urina tem de ser grande, uma vez que pequenas quantidades podem indicar outros problemas fisiológicos.

A enurese é uma das doenças mais frequentes e crônicas da infância com prevalência de aproximadamente $10 \%$ entre as crianças de sete anos de idade (Nevéus, 2009). No Brasil, estudo recente desenvolvido por Mota, Barros, Matijasevich, e Santos (2015) avaliou a prevalência de enurese em 3.602 crianças com idade de sete anos pertencentes a coorte de nascimentos iniciada em 2004. Os resultados, apontaram uma prevalência de 10,6\%, sendo $11,7 \%$ meninos e $9,3 \%$ meninas.

Várias etiologias têm sido propostas para a enurese, mas o reconhecimento da heterogeneidade de apresentações clínicas sugere influência multifatorial, no entanto, sabe-se hoje que, na grande maioria dos casos, os determinantes biológicos têm maior influência no aparecimento da enurese em comparação a determinantes ontogenéticos ou culturais (APA, 2013; Nevéus et al., 2006).

Entre os determinantes biológicos destacam-se: 1. dificuldade de despertar ao sinal de bexiga cheia; 2. poliúria noturna (falha na liberação do hormônio vasopressina que concentra a urina); e 3. atividade detrusora disfuncional (contrações não inibidas do músculo detrusor da bexiga). Butler e Holland (2000) chamam esse modelo de compreensão da enurese de três sistemas.

É importante esclarecer que a enurese não é consequência, e sim causa de problemas emocionais, como estresse, ansiedade e o medo (APA, 2013; Nevéus et al., 2006). Um estudo indicou que, em uma amostra de 149 crianças e adolescentes com enurese, 132 (89\%) sofreram algum tipo de agressão devido ao episódio de enurese (Sapi et al., 2009).

Adicionalmente, um estudo nacional identificou correlação positiva e estatisticamente significativa entre intolerância manifestada pelos pais diante da enurese e o impacto emocional sofrido pela criança (Ferrari, Alckmin-Carvalho, Silvares, \& Pereira, 2015). Esse achado indica que quando os episódios de molhar a cama são consequenciados com punição ou ameaças, os problemas psicológicos se manifestam. Além disso, quando os episódios de molhada acontecem na presença de outras crianças, é possível que haja risco aumentado para sofrer bullying, que é fator de risco para o surgimento de diversos problemas emocionais e comportamentais (AlckminCarvalho, Izbicki, \& Melo, 2014).

A enurese pode ser tratada por diferentes métodos. Entre as cinco modalidades (farmacológica: antidepressivos tricíclicos, oxibutinina e desmopressina, e não farmacológica: alarme e acupuntura) de tratamento para enurese noturna, o alarme é considerado a melhor opção, sendo recomendada pela ICSS como tratamento de primeira linha (Hjalmas et al., 2004). Trata-se de um dispositivo sonoro usado na roupa ou na cama da criança que é acionado pelo início da micção. A criança é acordada pela ativação do alarme, o que a leva, ao longo do tratamento, a acordar antes de iniciar a micção ou a passar a noite seca. Um dos processos pelo qual o tratamento parece funcionar é a esquiva ativa, em que a bexiga cheia assume caráter de estímulo discriminativo para o acionamento do alarme, fazendo com que a criança acorde ou contraia a musculatura pélvica para continuar a noite de sono.

A literatura (Butler \& Gasson, 2005; Perrin, Sayer, $\&$ While, 2015) aponta a ampla utilização do alarme em diversos países com índice de sucesso em torno de $65 \%$. Recente revisão sistemática (Perrin et al., 2015) investigou a eficácia da terapia por alarme vs por desmopressina no tratamento de enurese noturna. Os resultados mostraram que o alarme, a longo prazo, foi mais eficaz no tratamento de enurese noturna do que a desmopressina. Além de ter o efeito mais duradouro, entre os tratamentos para enurese, o alarme é o tratamento de mais baixo custo (Houts, 2003).

A presença de enurese está associada a diversas consequências psicológicas, repercutindo nos índices de problemas de comportamento de crianças e adolescentes nessa condição. Ferrari et al. (2015), avaliaram os indicadores de problemas de comportamento em uma amostra de 31 crianças com enurese que procuraram tratamento em uma clínica-escola. A média dos escores de problemas de comportamentos internalizantes, como depressão, ansiedade e isolamento, atingiram níveis clínicos.

A enurese afeta também a percepção que crianças e adolescentes têm de si mesmos (autoconceito) (Ozgür, Özgür, \& Örün, 2009). O autoconceito refere-se à percepção que o indivíduo tem sobre si mesmo, em diversas áreas de sua vida, como a pessoal, social, familiar e escolar. Trata-se de um construto multideterminado (determinado a partir da complexa interação entre diversas variáveis ambientais), contextual (varia de acordo com o ambiente em que é avaliado), e sempre em desenvolvimento (Sisto \& Martinelli, 2004). Outra característica do autoconceito é seu caráter de multidimensionalidade.

Embora autores utilizem autoestima e autoconceito como sinônimos (eg., Hugles, 1984), cabe fazer uma distinção: o autoconceito é um construto mais amplo que a autoestima, e avalia, além da percepção que o indivíduo tem sobre suas características, seu modo de ser e de agir (autoconceito pessoal, que seria equivalente à autoestima), a percepção sobre competências e habilidades no âmbito escolar, social e familiar.

Autores indicam quatro dimensões (tipos de autoconceito): pessoal (sentimentos que a pessoa tem com 
relação ao seu modo de agir e de ser em diferentes situações), social (percepção que o indivíduo tem sobre seu nível de aceitação social e sobre sua habilidade de estabelecer e manter vínculos afetivos), escolar (percepção que o indivíduo tem de suas habilidades acadêmicas e interpessoais na escola) e familiar (percepção que o indivíduo tem sobre sua adequação às demandas de seus pais, e sobre seu papel como filho) (Sisto \& Martinelli, 2004).

Nos últimos anos, os estudos começaram a focar na relação entre enurese e autoestima. Sobre esse aspecto, Koca et al. (2014) compararam a autoestima e os indicadores de depressão em uma amostra de 38 crianças com enurese noturna com outras 46 crianças controle. Os autores encontraram diferenças significativas entre os grupos, com índices mais elevados de depressão e mais baixos de autoestima nas crianças com enurese.

Resultado similar foi encontrado em outro estudo que comparou a autoestima de um grupo de crianças com enurese $(n=63)$ pareados por sexo e idade a outras 63 crianças controle. Os autores encontraram menores níveis de autoestima entre crianças com enurese nos domínios de autoimagem e de relacionamentos interpessoais, havendo diferenças estatisticamente detectáveis entre os grupos (Kanaheswari, Poulsaeman, \& Chandran, 2012). Na mesma direção, Collier, Butler, Redsell, e Evans (2002) encontraram, na avaliação de 114 crianças com enurese, comprometimento em termos de autoconceito.

Corroborando essa hipótese, o estudo randomizado controlado de Longstaffe, Moffatt, e Whalen (2000) investigou, se após seis meses de tratamento de alarme para o tratamento da enurese, o autoconceito das crianças modificaria. Os resultados do estudo demonstraram um efeito benéfico no autoconceito dos participantes, avaliado pela escala Piers-Harris Self-Concept Scale, após o tratamento via alarme.

A pesquisa de Hägglöf, Andrén, Bergström, Marklund, e Wendelius (1998) avaliou a autoestima de 66 crianças com enurese após o tratamento e as comparou com um grupo de 64 crianças controle. As crianças, nas quais o tratamento da enurese foi eficaz apresentaram melhora na autoestima avaliada pela escala sueca $I$ think I am, na avaliação de seguimento de seis meses. Os autores enfatizaram que crianças com enurese apresentam baixa autoestima e que a melhora desta pode ser alcançada com o tratamento para enurese.

Pesquisadores apontam que problemas de autoestima relacionados à enurese podem perdurar para além da infância e adolescência, mesmo nos casos em que há remissão espontânea (Dursun, Malkoc, Okcelik, Cirakoglu, \& Ates, 2014). Esse dado indica que a enurese, embora pareça não ser um indício de problemas psicológicos, pode precedê-los e ser fator de risco para seu aparecimento e manutenção, podendo afetar as relações sociais, o rendimento acadêmico, as relações amorosas e o rendimento no trabalho. Dessa forma, entende-se que o tratamento para enurese seria uma medida indireta de tratamento e de prevenção de problemas de autoconceito.

Embora a literatura nacional (Pereira, Costa, Rocha, Arantes, \& Silvares, 2009) aponte os efeitos do tratamento comportamental para enurese sobre os indicadores de problemas de comportamento, ainda não se tem estudo nacional publicado sobre o efeito do tratamento no autoconceito da criança ou adolescente.

O objetivo do presente estudo foi avaliar a eficácia do tratamento comportamental com alarme à distância para enurese e avaliar efeitos da intervenção no autoconceito dos participantes. Tem-se como hipótese que o tratamento da enurese com desfecho de sucesso produzirá melhoras nos indicadores de autoconceito das crianças e adolescentes.

\section{Método}

\section{Delineamento e Participantes}

Trata-se de um ensaio clínico com delineamento quase experimental de grupos. Participaram do estudo 31 crianças com diagnóstico de enurese primária monossintomática de acordo com os critérios da ICCS (ao menos um episódio por mês), atendidas em um serviço-escola de Psicologia de uma capital brasileira, e seus cuidadores. Foram adotados os seguintes critérios para a inclusão das famílias no tratamento: idade entre seis e 11 anos; apresentar enurese primária monossintomática de acordo com os critérios da ICCS; ter relatado realização de consulta médica prévia, a fim de excluir outras hipóteses diagnósticas e comparecer às duas sessões iniciais de atendimento e responder aos dois primeiros contatos telefônicos após entrega do alarme.

A idade dos participantes variou entre seis e 11 anos $(M=8,3 ; D P=1,3 ;$ IC 95\%: 7,84-8,76), sendo 15 do sexo masculino (48,3\%) e 16 do sexo feminino $(51,7 \%)$.

\section{Instrumentos}

Formulário de avaliação da enurese (Blackwell, 1989). Avalia dados relativos à enurese, como: história e tipo da enurese, tratamentos anteriores, atitudes parentais, condições de moradia, frequência e padrão dos episódios de enurese e histórico médico. É utilizado para diagnóstico e verificação da adequação aos critérios de inclusão.

Escala de Autoconceito Infanto Juvenil (Sisto \& Martinelli, 2004). Instrumento de autorrelato que permite avaliar o autoconceito da criança e do jovem, em distintos contextos sociais (pessoal, escolar, social, familiar e geral). É destinada para a faixa etária de 8 a 16 anos. São exemplos de afirmativas: "Sinto-me mais esquisito do que os outros"; "Considero-me o mais esperto da sala" e "Acho que sou muito preocupado". O sujeito indica, no questionário, o grau em que cada afirmativa se aplica a ele. As escalas são de cinco pontos, variando de "discordo totalmente" a "concordo totalmente". Pontuações altas indicam um autoconceito positivo. Não há ponto de corte para interpretação dos resultados. No 
presente estudo, foi comparada a média do grupo antes e após a intervenção.

Alarme de cabeceira para enurese (bedside alarm ou bell and pad). Semelhante ao modelo inglês, produzido pelo Laboratório de Terapia Comportamental do Instituto de Psicologia da Universidade de São Paulo. $\mathrm{O}$ equipamento consiste em duas partes: tapete plástico detector de urina e unidade de controle. $\mathrm{O}$ tapete deve ser colocado por baixo do lençol para que a urina a alcance rapidamente. Quando detectada a urina, o alarme é ativado para acordar a criança. Além do alarme sonoro, ascende-se uma luz para guiar a pessoa a encontrá-lo mesmo em locais escuros. O sistema é alimentado por uma bateria de $9 \mathrm{~V}$.

Questionário sociodemográfico. Elaborado pelos pesquisadores, compreende informações como idade, sexo, escolaridade e nível socioeconômico dos participantes.

Guia de pais para o tratamento da enurese. Baseado no instrumento de Houts (1990), contém instruções detalhadas para tratamento, tais como: horário de dormir, ingestão de líquidos, manejo do alarme e superaprendizagem, erros comuns observados no procedimento dos pais, recomendações sobre o manejo em cada situação, como treino de autocontrole, treino de limpeza e treino com o alarme e informações acerca de como proceder com possíveis problemas que possam surgir.

Registro de episódios da enurese. Registro dos dias e horários de ocorrência de molhada, tamanho da mancha de urina, se a criança acordou com o alarme sem ajuda dos pais e a quantidade de líquido ingerido no período de prevenção de recaída.

\section{Procedimentos}

Esta pesquisa foi aprovada pelo Comitê de Ética em Pesquisa com Seres Humanos da universidade em que foi realizado o estudo. Somente os participantes que assinaram o Termo de Consentimento foram avaliados, tendo sua identidade preservada e o direito de abandonar o estudo sem nenhum ônus. As famílias procuram a clínica para tratamento de enurese em fluxo contínuo. Para evitar a formação de uma fila de espera muito extensa, os participantes foram divididos em subgrupos. Dessa forma, ao acúmulo de dez famílias (cinco de cada grupo), elas eram chamadas para o início do tratamento, que consiste no comparecimento às sessões iniciais do programa, que correspondem a etapa de pré-intervenção. As famílias foram contatadas com quinze dias de antecedência ao primeiro encontro, a fim de realizarem um registro das molhadas neste período, que foi utilizado como linha de base.

\section{Pré-Intervenção}

As famílias interessadas em participar do atendimento para a enurese, após divulgação do serviço na mídia, entraram em contato com o pesquisador por telefone. Foram convidadas para o primeiro encontro, de triagem, realizado por duplas de estudantes de graduação em Psicologia e pós-graduação em Psicologia Clínica, todos vinculados ao grupo responsável por oferecer o atendimento a pacientes com enurese. $\mathrm{Na}$ triagem, foram aplicados os seguintes instrumentos: Formulário de avaliação da enurese, Escala de Autoconceito Infantojuvenil e questionário sociodemográfico.

As instruções para o tratamento de enurese foram estruturadas em duas sessões presenciais, realizadas com uma semana de intervalo, em horário que possibilitava a presença de todos os pais. Cada sessão teve uma hora de duração e foi realizada em grupo, sendo que na primeira metade a pesquisadora conversava com as crianças e na segunda metade conversava com os pais, enquanto as crianças ficavam em recreação com um estagiário. As sessões foram filmadas com o devido consentimento dos participantes.

As famílias que preencheram os critérios de inclusão foram convidadas a participar das sessões presenciais. Na primeira sessão, o programa de atendimento foi apresentado, e o aparelho de alarme teve seu funcionamento explicado. O Guia dos Pais e o Registro de Episódios da Enurese foram entregues. Os pais e as crianças foram orientados a discutir o tratamento em casa, fazer as alterações de hábito e de estrutura necessárias para o bom andamento do tratamento e a anotar possíveis dúvidas em relação ao procedimento.

Na segunda sessão, as dúvidas trazidas pelas famílias foram abordadas e foi recapitulado o funcionamento do alarme e dos outros procedimentos do tratamento. Os alarmes foram entregues e os horários do acompanhamento telefônico foram combinados.

\section{Acompanhamento Telefônico}

O acompanhamento do tratamento via telefone foi realizado quinzenalmente, por no máximo 28 semanas ou até que a criança preenchesse os critérios de alta (ICCS), e seguiu procedimento padronizado (Silvares, Pereira, \& Arantes, 2007): (a) levantamento das informações do Registro de Episódios da Enurese; (b) verificação do seguimento das instruções gerais, (c) interrogação dos motivos de não seguimento das instruções, tais como acordar a criança no meio da noite para levá-la ao banheiro, não usar o alarme, não ajudar a criança a levantar quando o alarme é disparado, (d) identificação e validação dos sinais de progressos, tais como diminuição do número de "molhadas" por noite, noites sem "molhadas", número de noites seguidas sem "molhadas"; (e) busca de soluções para as dificuldades e acompanhamento das estratégias propostas nas sessões subsequentes; (f) discussão buscando a identificação de fatores que, em cada caso individual, poderiam facilitar o seguimento dos procedimentos do alarme, como presença de luz acesa e dormir cedo; (g) acompanhamento de procedimentos adicionais, como o treino de autocontrole, treino de limpeza, técnicas de despertar e restrição da ingestão de líquidos em período noturno; (h) discussão de resistências 
ao uso do alarme apresentadas pelas crianças e manifestadas pelos pais e (i) providências caso fosse necessário realizar trocas no alarme.

\section{Pós-Intervenção}

Ao término do período de tratamento, cada uma das famílias foi convocada a comparecer ao Instituto de Psicologia da Universidade onde foi realizada a pesquisa, para devolver o alarme, preencher novamente Escala de Autoconceito Infantojuvenil e entregar os Registros de episódios da enurese para conferência.

\section{Análise dos Dados}

Foram realizadas análises descritivas (proporções, médias, desvios padrões e intervalo de confiança). Comparações entre a etapa pré e pós-intervenção foram realizadas por meio do teste $t$, seguido pelo cálculo da magnitude do efeito $(d)$ quando estatisticamente significativo. A magnitude do efeito foi estimada como a diferença entre as médias dos grupos dividida pelo desvio padrão comum. Convencionalmente, um valor de $d=0,20$ representa uma magnitude de efeito pequeno, $d=0,50$ indica magnitude média e $d=0,80$ indica uma magnitude de efeito elevada (Cohen, 1988).

Para analisar as diferenças entre os participantes quanto ao autoconceito, a amostra foi dividida em dois grupos considerando o sucesso (criança obter 14 noites secas consecutives) e o insucesso (criança não obter 14 noites secas consecutivas durante as 28 semanas ou abadono da intervenção) no tratamento. Foram realizadas comparações referentes ao momento pré-tratamento para os grupos sucesso e insucesso em relação ao sexo por meio do teste exato de Fisher e em relação a idade por meio do teste de Mann-Whitney.

Ao dividir os grupos, a análise gráfica e o teste ShapiroWilk indicaram que a distribuição dos dados não obedeceu aos critérios de normalidade, de modo que foram utilizados testes estatísticos não-paramétricos. Por esta razão foi utilizado o teste Mann-Whitney para avaliar as variações do autoconceito considerando a etapa da pesquisa (pré e pós) e o desfecho (sucesso e insucesso). Adotou-se nível de probabilidade de $95 \%(p<0,05)$ para a rejeição das hipóteses de nulidade. Os dados foram tabulados e analisados através do software SPSS 17.0 para Windows.

\section{Resultados}

Os resultados demonstraram que a maior parte das crianças $(n=22,71 \%)$ ficou 14 noites sem molhar a cama, obtendo sucesso no tratamento. Três famílias desistiram do tratamento $(9,7 \%)$ e seis crianças $(19,3 \%)$ não atingiram o critério de sucesso, mesmo realizando o tratamento adequadamente. Os grupos sucesso e insucesso no tratamento não apresentaram diferenças estatisticamente significativas em relação a idade $(p=0,68)$ e sexo $(p=0,32)$ no momento prévio à intervenção.

Os dados referentes ao autoconceito foram avaliados em 18 crianças, uma vez que as outras 13 não estavam na faixa etária compreendida pelo Escala de Autoconceito Infantojuvenil, único instrumento validado disponível para avaliar autoconceito quando da execução do estudo. A Tabela 1 a seguir apresenta a variação das médias do autoconceito geral e em cada um dos domínios, em pré e pós intervenção.

Tabela 1

Variação da Média de Autoconceito em Pré e Pós Intervenção $(n=18)$

\begin{tabular}{|c|c|c|c|c|c|c|c|c|c|}
\hline \multirow[b]{2}{*}{ Autoconceito } & \multicolumn{3}{|c|}{ Pré-intervenção } & \multicolumn{3}{|c|}{ Pós-intervenção } & \multirow{2}{*}{$\begin{array}{c}\text { Variação } \\
(\%)\end{array}$} & \multirow[t]{2}{*}{ Valor $p^{*}$} & \multirow[t]{2}{*}{$d$} \\
\hline & Média & $D P^{* *}$ & IC $(95 \%)^{* * *}$ & Média & $D P$ & IC (95\%) & & & \\
\hline Pessoal & 4,8 & 1,9 & {$[4,1-5,4]$} & 6,1 & 1,9 & {$[5,4-6,7]$} & 54,40 & 0,02 & 0,68 \\
\hline Escolar & 4,3 & 1,6 & {$[3,7-4,8]$} & 3,8 & 1,7 & {$[3,2-4,4]$} & $-4,60$ & 0,146 & - \\
\hline Social & 9,7 & 1,7 & {$[9,1-10,3]$} & 10 & 1,8 & {$[9,3-10,6]$} & 6,40 & 0,213 & - \\
\hline Familiar & 5,6 & 1,3 & {$[5,1-6,0]$} & 5,5 & 2,5 & {$[4,6-6,3]$} & $-1,30$ & 0,197 & - \\
\hline Geral & 24,4 & 3,6 & {$[23,1-25,6]$} & 25,4 & 4,0 & {$[23,9-26,8]$} & 5,00 & 0,224 & - \\
\hline
\end{tabular}

Nota. ${ }^{*}$ Teste t de Student; ${ }^{* *} \mathrm{DP}=$ Desvio Padrão; ${ }^{* * *} \mathrm{IC}=$ Intervalo de Confiança

Houve diferença estatisticamente significativa na variação da média de autoconceito pessoal em pré e pós intervenção $(4,8 ; D P=1,9 ;$ IC 95\%: 4,1-5,4 vs 6,1; $D P=1,9 ;$ IC 95\%: 5,4-6,7), sendo a variação dos escores de $54,4 \%$, independentemente do desfecho do tratamento. $\mathrm{O}$ valor $d$ de médio a alto, indica que a diferença entre os escores médios dos períodos pré e pós são substanciais.
Para autoconceito geral, familiar, social e escolar houve pequenas variações, para mais ou para menos, sem diferenças significativas. Na tabela 2, está apresentada a variação da média de autoconceito a partir do desfecho do tratamento (sucesso ou fracasso) no período pré e pós-intervenção. 
Tabela 2

Variação da Média de Autoconceito versus Resultado do Tratamento $(n=18)$

\begin{tabular}{|c|c|c|c|c|c|c|c|}
\hline \multirow{2}{*}{ Autoconceito } & \multirow{2}{*}{ Etapa } & \multicolumn{3}{|c|}{ Participantes Insucesso $(n=5)$} & \multicolumn{3}{|c|}{ Participantes Sucesso $(n=13)$} \\
\hline & & Média & $\mathrm{DP}$ & $p$ & Média & $D P$ & Valor $p^{*}$ \\
\hline \multirow{2}{*}{ Pessoal } & PRÉ & 4,4 & 1,5 & \multirow{2}{*}{0,641} & 4,9 & 2,0 & \multirow{2}{*}{0,610} \\
\hline & PÓS & 5,6 & 2,0 & & 6,3 & 2,0 & \\
\hline \multirow{2}{*}{ Escolar } & PRÉ & 3,6 & 2,0 & \multirow{2}{*}{0,401} & 4,6 & 1,5 & \multirow{2}{*}{0,387} \\
\hline & PÓS & 3,2 & 1,6 & & 4 & 1,7 & \\
\hline \multirow{2}{*}{ Social } & PRÉ & 9,4 & 1,1 & \multirow{2}{*}{0,441} & 9,8 & 1,9 & \multirow{2}{*}{0,03} \\
\hline & PÓS & 8,6 & 2,0 & & 10,5 & 1,5 & \\
\hline \multirow{2}{*}{ Familiar } & PRÉ & 5 & 1,2 & \multirow{2}{*}{0,581} & 5,8 & 1,4 & \multirow{2}{*}{0,241} \\
\hline & PÓS & 5,4 & 3,5 & & 5,5 & 1,5 & \\
\hline \multirow{2}{*}{ Geral } & PRÉ & 22,4 & 2,5 & \multirow{2}{*}{0,069} & 25,2 & 3,8 & \multirow{2}{*}{0,094} \\
\hline & PÓS & 22,8 & 3,2 & & 26,4 & 4,0 & \\
\hline
\end{tabular}

Nota. ${ }^{*}$ Teste de Mann Whitney

Houve diferença estatisticamente significativa entre as médias de pré e pós-intervenção no autoconceito social das crianças que obtiveram sucesso no tratamento $(9,8 ; D P=1,9$ vs 10,$5 ; D P=1,5 ; p=0,03)$, indicando melhora da média dos escores nesse domínio. Não foram verificadas outras variações significativas entre as médias dos escores em pré e pós-intervenção.

\section{Discussão}

Os resultados desta pesquisa indicam que o tratamento com alarme para enurese realizado a distância é eficaz, uma vez que a maior parte dos participantes (71\%) obtiveram sucesso de acordo com os critérios do ICCS. O alto índice de sucesso obtido na modalidade de acompanhamento do tratamento à distância, corrobora sua viabilidade previamente indicada por outros autores (Houts, 2003; Nevéus et al., 2010; Pereira, 2010).

$\mathrm{O}$ índice de $71 \%$ de sucesso no tratamento, verificado no presente estudo, é ligeiramente mais alto do que o encontrado na literatura, que aponta um índice em torno de 65\% (Butler \& Gasson, 2005; Perrin et al., 2015). Em um país de dimensões continentais, como o Brasil, a possibilidade de oferecer atendimento para enurese à distância diminui os custos da intervenção associados ao deslocamento das famílias até o centro de atendimento semanalmente, e possivelmente aumenta a aderência dos participantes. Uma vez que a intervenção à distância apresentou resultados satisfatórios, essa intervenção pode ser oferecida para famílias que tenham filhos com enurese de diversas regiões do país.

É importante destacar que na atual pesquisa, além dos participantes que completaram o tratamento sem obter sucesso, também foram incluídos na análise os participantes que desistiram do tratamento, dado que não costuma ser apresentado nesse tipo de estudo. Sustenta essa medida a compreensão de que a desistência da família é uma expressão de falha no tratamento.

Sobre a associação entre o tratamento e os indicadores de autoconceito das crianças, o efeito do tratamento do alarme repercutiu em aumento do autoconceito pessoal das crianças que participaram da pesquisa e no autoconceito social daquelas que tiveram sucesso no tratamento. Tais resultados vão ao encontro dos resultados obtidos por Longstaffe, Moffat, e Whalen (2000), que reportaram um autoconceito mais positivo em crianças que alcançam resultados satisfatórios no tratamento para enurese.

Diferentemente dos estudos de Häagglöf, et al. (1998), Longstaffe et al. (2000) e de Nawaz, Griffiths e Tappin (2002) que demonstraram a melhora da autopercepção dos participantes apenas depois de seis meses após o termino do tratamento, isto é, no período de seguimento, este estudo demonstrou o aumento nos escores de autoconceito na avaliação realizada pós-tratamento, ou seja, assim que terminou a intervenção. A melhora nos domínios pessoal e social da autopercepção da criança ocorreu de forma concomitante à redução de molhadas. Sobre este aspecto, acredita-se que uma vez que a enurese causa impacto no autoconceito das crianças, é de se esperar que a sua resolução traga melhoras nesses indicadores.

Em suma, os resultados deste estudo respondem aos objetivos propostos ao demonstrarem um índice alto de sucesso do tratamento para enurese via alarme na modalidade de acompanhamento à distância. Tais resultados são positivos para a ampliação de acesso aos benefícios do tratamento também para aqueles que apresentam dificuldade em comparecer a sessões presenciais. Além disso, este estudo evidencia a associação entre enurese e autoconceito, de modo que o sucesso no tratamento da enurese está associado ao aumento no autoconceito pessoal e social dos participantes. 
Embora os objetivos tenham sido alcançados faz-se necessário apontar algumas limitações do estudo, como o pequeno número de participantes que foram avaliados em termos de autoconceito. A perda de 13 crianças, que tinham menos de oito anos de idade (faixa não coberta pelo Escala de Autoconceito Infanto-Juvenil) fragiliza a generalização dos resultados encontrados para outras crianças.

Embora tenha sido realizadas comparações entre os grupos sucesso e insucesso no tratamento no momento prévio a intervenção nas variáveis idade e sexo, devido à falta de informações, não foi possível realizar comparações em relação a outras variáveis, tais como renda e escolaridade da família, o que não permitiu verificar se nessas variáveis os grupos eram equivalentes. Além disso, o número reduzido de participantes não permitiu análises paramétricas que pudessem comparar variáveis dos participantes que tiveram e que não tiveram sucesso no tratamento, o que fortaleceria as evidências dos achados encontrados no presente estudo. Pesquisas adicionais, com uma amostra maior, e delineamento randomizado podem contribuir para produzir evidências de eficácia mais robustas.

Além disso, os escores de autoconceito foram obtidos exclusivamente por meio do autorrelato das crianças que compuseram a amostra. Especialmente entre crianças mais novas, que podem ter dificuldade de se avaliarem em termos subjetivos, futuras pesquisas que incluam outros informantes, como os pais, e professores, bem como a avaliação via entrevista clínica, podem ser úteis para investigar de forma mais abrangente o autoconceito da criança antes e depois do tratamento. Ademais, os autores apontam a necessidade do desenvolvimento e avaliação das evidências de validade de instrumentos que avaliem autoconceito na primeira infância. Pesquisas futuras são necessárias também para avaliar a manutenção dos resultados em períodos de seguimento. Espera-se que este estudo sirva de incentivo para pesquisas nacionais sobre o tema, elucidando a importância do tratamento, não somente para soluções da queixa da enurese, como também para seus efeitos positivos em termos de autopercepção.

\section{Referências}

American Psychiatric Association. (2013). The Diagnostic and Statistical Manual of Mental Disorder (DSM 5). Washington D.C.

Arantes, M. C. (2007). Problemas de comportamento e resultados do tratamento com alarme para enurese primária. (Dissertação de Mestrado). Universidade de São Paulo, Instituto de Psicologia, São Paulo.

Alckmin-Carvalho, F., Izbicki, S., \& Melo, M. H. S. (2014). Problemas de comportamento segundo vítimas de bullying e seus professores. Estudos e Pesquisas em Psicologia 14(3), 834-853.

Blackwell, C. (1989). A Guide to enuresis: A guide to a treatment of enuresis for professionals. Bristol: Enuresis Resource and Information Center.

Butler, R. J., \& Gasson, S. L. (2005). Enuresis alarm treatment. Scandinavian Journal of Urology and Nephrology, $39(5), 349-357$. doi:10.1080/00365590500220321

Cohen, J. (1988). Statistical power analysis for the Behavioral Sciences. Hilsdale, NJ: Erlbaum.

Collier, J., Butler, R. J, Redsell, A., \& Evans, J. H. C. (2002). An investigation of the impact nocturnal enuresis on Children's Self-Concept. Scandinavian Journal of Urology and Nephrology, 36(3), 204-208. doi:10.1080/003655902320131884

Dursun, F., Malkoc, E., Okcelik, S., Cirakoglu, A., \& Ates, F. (2014). The Effect of Nocturnal Enuresis in Adults on Sexual Satisfaction and Self-Esteem. Andrology, 3(118), 2167-0250. doi:10.4172/2167-0250.1000118

Ferrari, R. A. (2014). Acompanhamento à distância do tratamento com alarme para enurese: efeitos dos problemas de comportamento. (Dissertação de Mestrado). Instituto de Psicologia da Universidade de São Paulo.

Ferrari, R. A., Alckmin-Carvalho, F., Silvares, E. F. D. M., \& Pereira, R. F. (2015). Enurese noturna: associações entre gênero, impacto, intolerância materna e problemas de comportamento. Psicologia: Teoria e Prática, 17(1), 85-96. doi: 10.15348/1980-6906

Häagglöf, B., Andrén, O., Bergström, E., Marklund, L., \& Wendelius, M. (1998). Self-esteem in children with nocturnal enuresis and urinary incontinence: Improvement of self-esteem after treatment. European Urology, 33(3), 16-19. doi: 10.1159/000052236

Houts, A. C. (1990). Guia dos pais para o tratamento da enurese. Traduzido e adaptado pelo Projeto Enurese, Instituto de Psicologia, Universidade de São Paulo, SP.

Houts, A. C. (2003). Behavioral treatment for enuresis. In A. E. Kazdin, J. R. Weisz, (Orgs.). Evidence-based Psychotherapies for Children and Adolescents (pp. 389-406). New York: The Guilford Press.

Hughes, H. M. (1984). Measures of self-concept and self-esteem for children ages 3-12 years: A review and recommendations. Clinical Psychology Review, 4(6), 657-692. doi:10.1016/0272-7358(84)90011-4

Kanaheswari, Y., Poulsaeman, V., \& Chandran, V. (2012). Self-esteem in 6 to 16 year olds with monosymptomatic nocturnal enuresis. Journal of Pediatrics and Child Health, 48(10), 178-182. doi: 10.1111/j.1440-1754.2012.02577.x

Koca, O., Akyüz, M., Karaman, B., Özcan, Z. Y., Öztürk, M., Sertkaya, Z., \& Karaman, M. I. (2014). Evaluation of depression and selfesteem in children with monosymptomatic nocturnal enuresis: A controlled trial. Archivio Italiano di Urologia e Andrologia, 86(3), 212-214. doi: 10.4081/aiua.2014.3.212

Longstaffe, S., Moffatt, M., \& Whalen, J. (2000). Behavioral and self-concept changes after six months of enuresis treatment: A randomized, controlled trial. Pediatrics, 105(4), 935-940.

Mota, D. M., Barros, A. J., Matijasevich, A., \& Santos, I. S. (2015). Prevalence of enuresis and urinary symptoms at age 7 years in the 2004 birth cohort from Pelotas, Brazil. Jornal de Pediatria, 91(1), 52-58. doi:10.1016/j.jped.2014.04.011 
Nawaz, S., Griffiths, P., \& Tappin, D. (2002). Parent-administered modified dry-bed training for childhood nocturnal enuresis: Evidence for superiority over urine-alarm conditioning when delivery factors are controlled. Behavioral Interventions, 17(4), 247-262. doi: 10.1002/ bin. 120

Nevéus, T. (2009). Diagnosis and management of nocturnal enuresis. Current Opinion in Pediatrics, 21(2), 199-202. doi: 10.1097/ MOP.0b013e3283229b12

Nevéus, T., Gontard, A., Hoebeke, P., Hjälmås, K., Bauer, S., Bower, W., Jørgensen, T. M., Rittig, S., Walle, J. V., Yeung, C., \& Djurhuus, J. C. (2006). The standardization of terminology of lower urinary tract function in children and adolescents: Report from the Standardisation Committee of the International Children's Continence Society. The Journal of Urology, 176(1), 314-324. doi:10.1016/ S0022-5347(06)00305-3

Özgür, B. C., Özgür, S., Dogan, V., \& Örün, U. A. (2009). The efficacy of an enuresis alarm in monosymptomatic nocturnal enuresis. Singapore Medical Journal, 50(9), 879-880.

Pereira, R. F., Costa, N. J. D., Rocha, M. M., Arantes, M. C., \& Silvares, E. F. M. (2009). O efeito da terapia comportamental para enurese sobre outros problemas de comportamento. Psicologia: Teoria e Pesquisa, 25(3), 419-423.

Perrin, N., Sayer, L., \& While, A. (2015). The efficacy of alarm therapy versus desmopressin therapy in the treatment of primary monosymptomatic nocturnal enuresis: a systematic review. Primary Health Care Research \& Development, 16(1), 21-31. doi: 10.1017/ S146342361300042X

Report of the Expert Committee on the International Classification of Diseases (1984) - 10th revision: first meeting. Geneva: World Health Organization.

Sapi, M. C., Vasconcelos, J. S., Silva, F. G., Damião, R., \& Silva, E. A. D. (2009). Assessment of domestic violence against children and adolescents with enuresis. Jornal de Pediatria, 85(5), 433-437. doi: S0021-75572009000500011

Sisto, F. F., \& Martinelli, S. C. (2004). Escala de Autoconceito Infanto-Juvenil (EAC-IJ). São Paulo: Vetor Editora.

\section{Sobre os autores}

Rafaela Almeida Ferrari é psicóloga formada pela Universidade de Mogi das Cruzes e tem mestrado realizado no Departamento de Psicologia Clínica da Universidade de São Paulo.

Rodrigo Fernando Pereira é psicólogo formado pela Universidade Presbiteriana Mackenzie e tem pós-doutorado realizado no Departamento de Psicologia Clínica da Universidade de São Paulo.

Felipe Alckmin-Carvalho é psicólogo formado pela Universidade Presbiteriana Mackenzie, mestre e doutorando pelo Departamento de Psicologia Clínica da Universidade de São Paulo.

Renatha Rafihi-Ferreira é psicóloga formada pela Universidade Paulista, Mestre em Análise do Comportamento pela Universidade Estadual de Londrina e Doutora pelo Departamento de Psicologia Clínica da Universidade de São Paulo. Atualmente realiza estágio de pósdoutorado pelo Departamento de Psicologia Clínica da Universidade de São Paulo, com bolsa FAPESP.

Edwiges Ferreira de Mattos Silvares é Professora Sênior do Departamento de Psicologia Clínica do Instituto de Psicologia da Universidade de São Paulo. Bolsista Produtividade CNPq 1B. 